\title{
Integrated analysis of different mRNA and miRNA profiles in human hypopharyngeal squamous cell carcinoma sensitive and resistant to chemotherapy
}

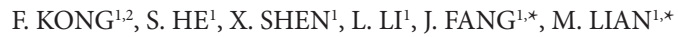 \\ ${ }^{1}$ Department of Otorhinolaryngology Head and Neck Surgery, Beijing Tongren Hospital, Capital Medical University, Beijing 100730, China; \\ ${ }^{2}$ Department of Otorhinolaryngology, The Hospital of Shunyi District Beijing, Beijing 101300, China \\ *Correspondence: fangjugao19651110@163.com; lianmeng19861222@163.com
}

Received March 20, 2019 / Accepted July 17, 2019

\begin{abstract}
The purpose of this study was to identify potential miRNAs and mRNAs involved in chemotherapy insensitivity in hypopharyngeal squamous cell carcinoma (HSCC) and to explore the underlying mechanisms involved to provide diagnostic markers and therapeutic targets for HSCC. We used microarrays to identify differences in both the mRNA and miRNA expression profiles between a group (twelve patients) sensitive to chemotherapy and a resistant group (nine patients). We then employed bioinformatics tools to examine the functions and pathways involved. The genes and miRNAs most related to chemotherapy sensitivity in HSCC were screened. Finally, a miRNA-mRNA-phenotype network was constructed with an integrated analysis based on the identified miRNAs and mRNAs. Nine differentially expressed miRNAs and one hundred differentially expressed mRNAs were identified, and the functions of these genes and miRNAs were predicted. Bioinformatics analysis revealed a regulatory network consisting of eight genes and two miRNAs that influenced HSCC chemosensitivity. According to our analysis, CCL4L1 may be a potential molecular marker for HSCC chemotherapy, and excess CCL4L1 leads to the upregulation of PRAME and the downregulation of miR-375, thus decreasing HSPB8 expression and promoting chemotherapy sensitivity. Our work provides reliable data for further studies investigating the mechanism of HSCC chemotherapy sensitivity.
\end{abstract}

Key words: hypopharyngeal squamous cell carcinoma, chemosensitivity, differentially expressed gene, microRNA, network

Hypopharyngeal squamous cell carcinoma (HSCC) is one of the most common head and neck squamous cell carcinomas, accounting for more than 160,000 new cases and 83,000 deaths annually $[1,2]$ and for $2-6 \%$ of all head and neck cancers $[3,4]$. In Europe and the United States, HSCC has been ranked as one of the most common human malignant tumors [5]. High risks of metastasis to cervical lymph nodes and a lack of evident clinical symptoms make HSCC one of the most difficult challenges in human malignancies [6-8]. It is urgent to explore novel effective methods for diagnosing and treating HSCC.

Despite advances in surgery and novel treatments such as chemotherapy and radiation therapy, the survival rate of latestage HSCC patients has not significantly improved [9]. The 5 -year survival rate of patients with advanced-stage HSCC is approximately $30-40 \%$ [10]. One of the important reasons for the unsatisfactory outcome is the occurrence of chemotherapy resistance. Although chemotherapy is an adjuvant regimen in combination with surgery or radiotherapy, it plays a crucial role in advanced or metastatic tumors, such as HSCC, particularly for the reason of organ preservation [11]. Many studies have demonstrated that chemotherapy, including docetaxel $(\mathrm{T})$, cisplatin $(\mathrm{P})$, and 5-fluorouracil $(\mathrm{F})$ is the most effective therapeutic management in HSCC [12]; nonetheless, the efficacy of chemotherapy is sometimes determined by the heterogeneity between individuals [13-14]. Moreover, the excessive application of chemotherapeutic agents can result in a series of adverse effects, including systemic toxicities and chemotherapy resistance. With developments in technology, genome-wide analyses based on microarray data are being increasingly used for some intractable issues due to their efficiency, versatility, and high-throughput nature [15]; to some extent, the expression of large gene sets is more capable of explaining a patient's response to a given therapeutic regimen [16]. Thus far, studies focusing on gene alterations involved in chemotherapy resistance in HSCC 
are still elusive and inadequate. In addition, previous studies have shown that the heterogeneity of HSCC in the intrinsic subtypes and epigenetic signatures is nonnegligible, which makes developing molecular markers for HSCC patient outcomes and the treatment response especially challenging. Microarray analysis is able to identify the overall differences in expression profiles between patients sensitive and resistant to chemotherapy on a large scale. A microarray can be used not only to determine the mechanism and key molecules of chemotherapy resistance but also to design personalized chemotherapy according to the patient.

In this study, we carried out a series of analyses to discover clinically relevant gene alterations by performing miRNA and mRNA microarrays, explored the miRNA and mRNA expression profiles in HSCC sensitive and resistant to chemotherapy, and constructed a miRNA-mRNA network by an integrated analysis. It is very promising to translate identified genes into molecular characteristics for targeted therapy and response assessment, which will ultimately accelerate personalized treatment.

\section{Materials and methods}

Patients and specimen selection. We collected HSCC tissue specimens from 21 patients (obtained from the Department of Head \& Neck Surgery, Beijing Tongren Hospital), including 9 tissue specimens from patients with resistant carcinoma and 12 tissue specimens from patients with sensitive HSCC. Information on the patients is shown in Table 1.

Table 1. Patient information used for analyses.

\begin{tabular}{lccccc}
\hline Sample & Sex & Age & TNM & Grade & Sensitivity \\
\hline BWS & male & 69 & T4aN2M0 & G2 & yes \\
FYY & male & 62 & T4aN2M0 & G1 & yes \\
YZL & male & 69 & T4N1M0 & G3 & yes \\
XZP & male & 49 & T3N2M0 & G2 & yes \\
LJ & male & 60 & T4bN2M0 & G2 & yes \\
LPL & male & 69 & T4aN0M0 & G2 & yes \\
LXJ & male & 44 & T2N2M0 & G3 & yes \\
GBM & male & 53 & T4aN0M0 & G1 & yes \\
ZJH & male & 49 & T4aN2M0 & G2 & yes \\
SB & male & 44 & T4aN2M0 & G3 & yes \\
ZSY & male & 60 & T3N1M0 & G2 & yes \\
LFL & male & 48 & T4bN2M0 & G1 & yes \\
DXX & male & 65 & T4bN2M0 & G1 & no \\
LGZ & male & 45 & T2N3M0 & G2 & no \\
YYT & male & 57 & T4bN3M1 & G1 & no \\
FKY & male & 69 & T3N2M0 & G1 & no \\
WZM & male & 71 & T4aN2M0 & G3 & no \\
NSS & male & 43 & T4bN2M1 & G3 & no \\
HYW & male & 69 & T2N1M0 & G1 & no \\
MH & male & 71 & T4aN0M0 & G1 & no \\
FWY & male & 43 & T4aN2M0 & G2 & no \\
\hline & & & & &
\end{tabular}

All patients received two periodic chemotherapies induced by TPF (taxane/cisplatin/5-FU). These HSCC pathological tissues were collected after resection during surgery. Each sample was immediately snap frozen in liquid nitrogen and stored at $-80^{\circ} \mathrm{C}$.

RNA extraction, cDNA synthesis and in vitro transcription. mRNA was extracted from tissue samples using TRIzol (Invitrogen), and then RNA quantity was examined by denaturing gel electrophoresis, which revealed at least two distinct bands representing $28 \mathrm{~S}$ and $18 \mathrm{~S}$ ribosomal RNA, suggesting no DNA contamination or RNA degradation. Total RNA was divided into two parts and used for separate miRNA and mRNA microarrays. First, reverse transcription was used to synthesize the first-strand cDNA, and secondstrand CDNA synthesis was used to convert single-stranded cDNA into double-stranded DNA with a PrimeScript ${ }^{\mathrm{tm}}$ Double Strand cDNA Synthesis Kit (TAKARA). Second, after purification by removing RNA, primers, enzymes, etc., the double-stranded DNA was used as a template for the transcription of biotinylated cRNA in vitro. Finally, the biotinylated cRNA was purified and prepared for hybridization with a prepared microarray. For the miRNA microarray, miRNAs were labeled using a miRNA Complete Labeling and Hyb Kit (Agilent Technologies, Santa Clara, CA, USA), and then hybridization was carried out on a human miRNA $\left(8^{\star} 60 \mathrm{~K}\right)$ v19.0 array in a hybridization oven (Agilent).

mRNA and miRNA expression profiles of HSCC. For the mRNA expression profile of HSCC, the Illumina Human HT-12 Bead Chip was applied for hybridization with the labeled cRNA. There are six types of internal parameters and 887 probes in this microarray for the quality control of all samples. Briefly, the cRNA samples were hatched with the Illumina Human HT-12 Bead Chip at room temperature and subjected to high temperature washes, ethanol washes and three washes at room temperature. After desiccation, images were collected with Illumina Bead Chip Reader software. Illumina Genome studio-Gene Expression software was employed to filter background noise and the missing value effect in the raw data. The quantile method was used for normalization. The gene expression profile was obtained using Illumina Custom software.

For the miRNA expression profile of HSCC, an Agilent miRNA Complete Labeling and Hybridization Kit (Agilent Technologies, USA) was used to conduct miRNA labeling and hybridization. The chip is able to explore 1205 human miRNAs and 144 human virus miRNAs from the Sanger database (Version 16.0). An Agilent scanner was used to scan the hybridized chip signal, and the raw data were normalized with GeneSpring GX software.

The identification of differentially expressed genes. The differentially expressed genes (DEGs) and differentially expressed miRNAs (DE-miRNAs) between sensitive and resistant HSCC specimens were determined by using the linear models for microarray data (limma) package in $\mathrm{R}$ [18], which adopts the empirical Bayesian theory to evaluate a 
standard error and exhibits excellent performance for small sample sets. The adjusted p-value was used to ensure statistical accuracy. A cluster analysis was performed to analyze the distribution of the DEGs and DE-miRNAs between the two groups. The nonparametric multivariate variance test was used to distinguish between groups based on distance matrices [19]. Nonmetric multidimensional scaling (NMDS) is a data analysis method that simplifies the research objects (samples or variables) of a multidimensional space to a low-dimensional space for positioning, analysis, and classification while retaining the original relationship between objects. According to the species information contained in the sample, the relationship is reflected in the multidimensional space in the form of points, while the difference

A
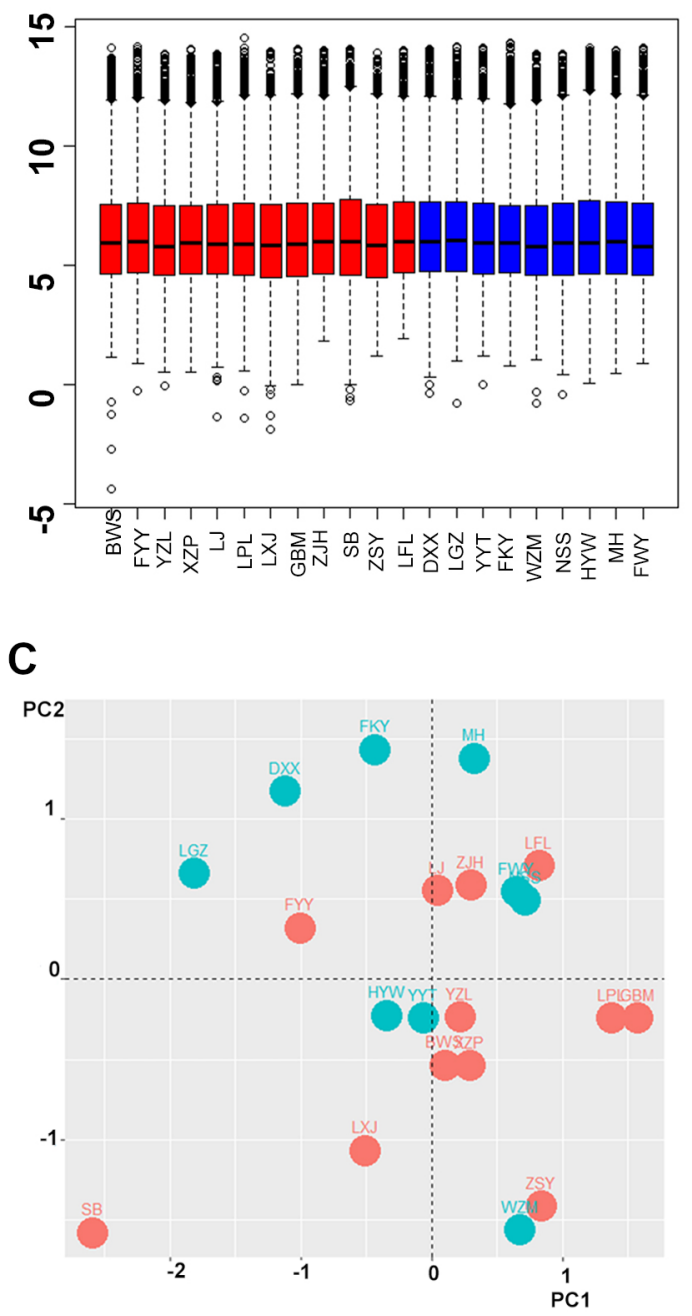

degree between different samples is reflected by the distance between points, and the spatial locus map of the sample is finally obtained. NMDS analysis (vegan package, bray distance algorithm) can visually show the degree of difference between different samples by the distance from a point to point; the more similar the expression of the DEG is between samples, the closer the sample points are [20].

Core genes and core miRNAs involved in the induction of chemosensitivity. The identified DEGs and DE-miRNAs were considered candidates for further integrative analysis. A Bayesian network was constructed to recognize the core genes associated with the induction of chemosensitivity among the identified candidate genes. The Bayesian network is a probabilistic graphical model (a type of statistical

B
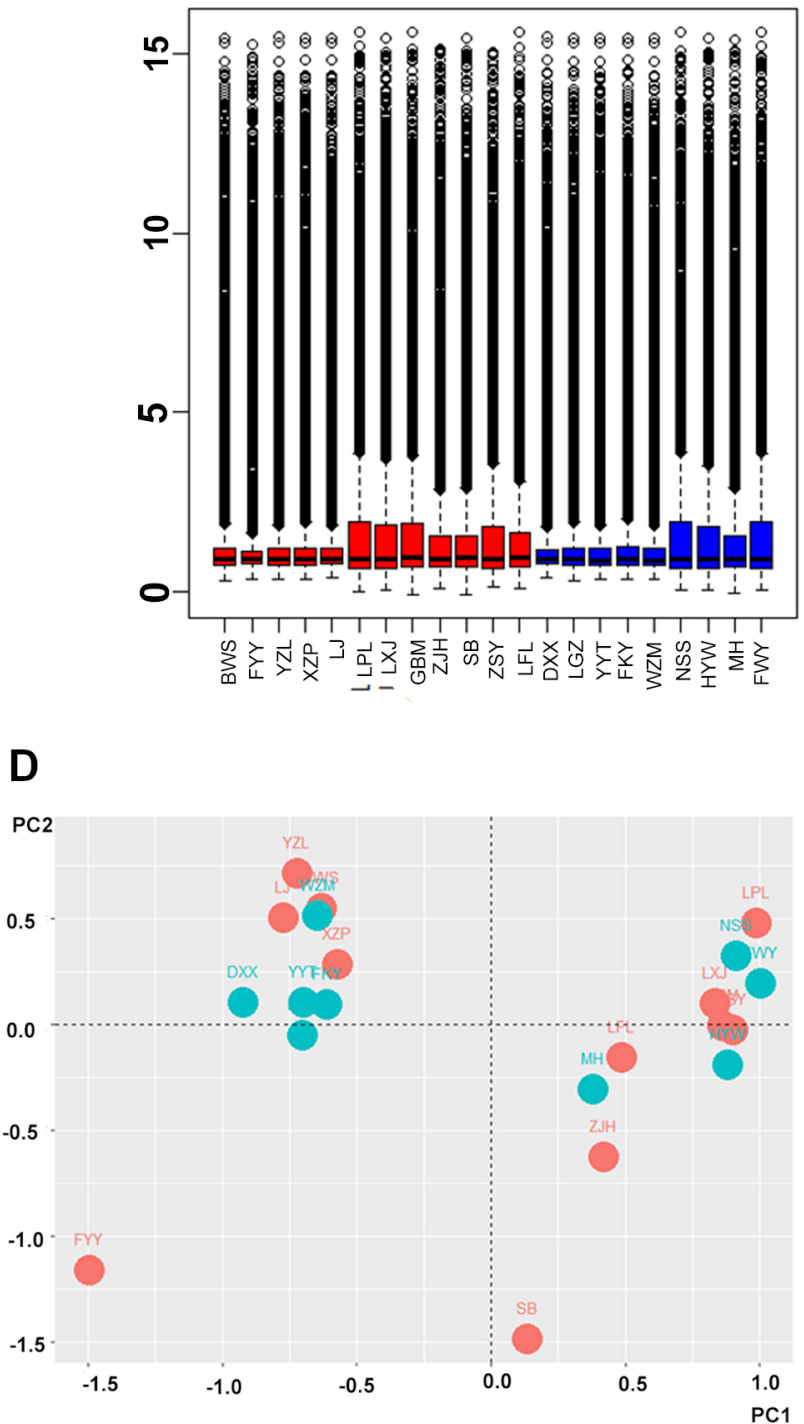

Figure 1. Sequencing quality control. The quality analysis of sequencing data of the mRNA microarray (A) and the miRNA microarray (B) showed that all samples basically followed the normal distribution, and the mean was equal, suggesting that the samples were comparable. NMDS analysis showed a strong correlation between the mRNA (C) and miRNA (D) expression profiles and phenotype. 
Table 2. The top 10 up- and downregulated genes between sensitive and resistant to chemotherapy HSCC samples.

\begin{tabular}{lccccc}
\hline mRNA & $\begin{array}{c}\text { Ave. } \\
\text { Expr. }\end{array}$ & $\mathbf{t}$ & logFC & p-value & adj.p-value \\
\hline GPC3 & 6.5256002 & 2.67312842 & 1.984141 & 0.0125 & 0.041948816 \\
HBB & 10.309794 & 2.40585216 & 1.782542 & 0.023128 & 0.045212163 \\
HBA2 & 10.148057 & 2.31728252 & 1.708241 & 0.028176 & 0.045212163 \\
FBN2 & 8.4282851 & 2.24686483 & 1.684762 & 0.032883 & 0.045212163 \\
HBA1 & 9.1824605 & 2.25917593 & 1.619915 & 0.032012 & 0.045212163 \\
CYP4F11 & 8.8518575 & 2.2641433 & 1.522736 & 0.031666 & 0.045212163 \\
PVALB & 5.0913239 & 2.37713763 & 1.465622 & 0.024666 & 0.045212163 \\
IL6 & 6.7520486 & 2.28776738 & 1.429614 & 0.030069 & 0.045212163 \\
GAGE7 & 4.4553063 & 2.18088574 & 1.321212 & 0.037926 & 0.045212163 \\
PRPH & 4.9360331 & 3.71769201 & 1.314387 & 0.000911 & 0.020068001 \\
LOC652694 & 9.709197 & -2.2420905 & -1.82363 & 0.033226 & 0.045212163 \\
AQP3 & 7.5347154 & -2.814754 & -1.85123 & 0.008924 & 0.041948816 \\
SFRP1 & 6.6736545 & -4.2041651 & -1.97475 & 0.00025 & 0.01447302 \\
SBSN & 10.203853 & -2.5251881 & -2.02226 & 0.017633 & 0.045212163 \\
LOC652102 & 6.4669035 & -2.5462251 & -2.02509 & 0.0168 & 0.045212163 \\
KRT78 & 6.8993388 & -2.8923161 & -2.11741 & 0.007399 & 0.041948816 \\
TCN1 & 7.2741944 & -2.5725616 & -2.12886 & 0.015808 & 0.045165714 \\
MAL & 8.4106509 & -2.9359099 & -2.80621 & 0.006654 & 0.041948816 \\
CRNN & 8.4505446 & -2.7259839 & -2.9542 & 0.011032 & 0.041948816 \\
KRT4 & 8.1869139 & -3.3672182 & -3.21512 & 0.002261 & 0.032590862 \\
\hline & & & & &
\end{tabular}
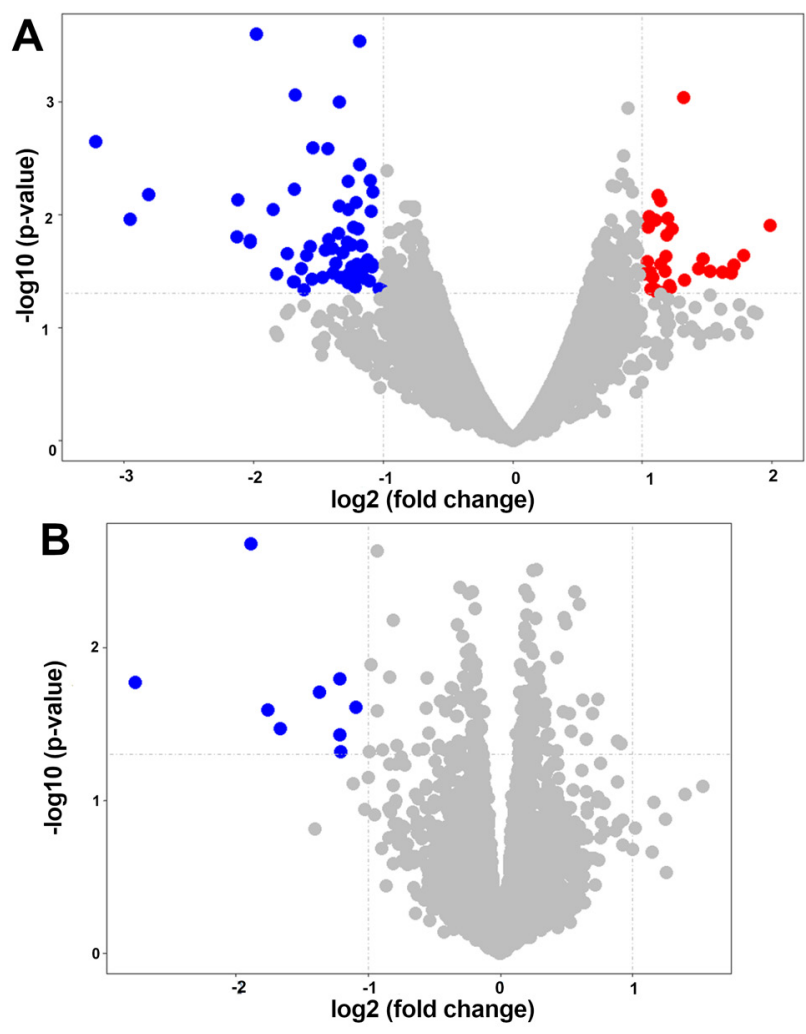

Figure 2. Expression levels of genes (A) and miRNAs (B) identified by the microarrays.
Table 3. Differentially expressed miRNAs between sensitive and resistant to chemotherapy HSCC samples.

\begin{tabular}{lccccc}
\hline miRNA & $\begin{array}{c}\text { Ave. } \\
\text { Expr. }\end{array}$ & $\mathbf{t}$ & $\operatorname{logFC}$ & p-value & $\begin{array}{c}\text { adj. } \\
\text { p-value }\end{array}$ \\
\hline hsa-miR-4448 & 4.22 & -3.51 & -1.89 & 0.0021 & 0.0189 \\
hsa-miR-3937 & 5.47 & -2.62 & -1.22 & 0.0160 & 0.0385 \\
hsa-miR-375 & 4.37 & -2.59 & -2.76 & 0.0170 & 0.0385 \\
hsa-miR-617 & 1.68 & -2.53 & -1.37 & 0.0196 & 0.0385 \\
hsa-miR-4462 & 4.34 & -2.42 & -1.09 & 0.0247 & 0.0385 \\
hsa-miR-650 & 3.95 & -2.40 & -1.76 & 0.0257 & 0.0385 \\
hsa-miR-31-5p & 11.61 & -2.27 & -1.67 & 0.0341 & 0.0420 \\
HBII-85-29 & 3.48 & -2.22 & -1.21 & 0.0373 & 0.0420 \\
hsa-miR-5580-3p & 1.79 & -2.10 & -1.21 & 0.0484 & 0.0484 \\
\hline
\end{tabular}

model) that represents a set of variables and their conditional dependencies via a directed acyclic graph (DAG). Bayesian network analysis performs three main inference tasks: inferring unobserved variables, parameter learning, and structure learning. The bnlearn $\mathrm{R}$ package was used in this study. The original data of the core microRNAs, core mRNAs, and disease phenotypes were discretized, and then Bayesian network structure learning (via constraint-based, score-based and hybrid algorithms), parameter learning (via maximum likelihood and Bayesian estimators) and approximate inference were carried out. As the coarse-grained reverse engineering of genetic regulatory networks, Bayes analysis can obtain ideal results with a global probability model [21]. In addition, it exhibits good ability in suggesting the causality of the core genes. Moreover, the accuracy of the identified core genes was further confirmed with random forests (RFs). The random forest package of R3.5.3 software was used to generate a random forest. Its data frame consists of different mRNAs (microRNAs) and tumor samples. A random forest is then adopted to reveal the supervised classification of samples, and the differentially expressed mRNAs (microRNAs) and core genes are sorted according to the mean decrease accuracy. This method is capable of classifying discrepant samples and providing variable importance scores of each gene for the classification [22].

\section{Results}

The identification of differentially expressed genes and miRNAs. Microarray technology was used to compare the miRNA and mRNA expression profiles between the two groups and to screen differentially expressed miRNAs as well as mRNAs. A total of 34,593 mRNAs and 6,077 miRNAs were detected (Table S1 and Table S2). After normalizing the gene expression profile data, all samples basically followed the normal distribution, and the mean was equal between samples, suggesting that the microarray data were reliable and could be compared between samples to identify the differentially expressed mRNAs and miRNAs 
(Figures 1A, 1B). NMDS analysis revealed a strong correlation between the mRNA or miRNA expression profile and phenotype, indicating a high success rate of screening genes or miRNAs relevant to the sensitivity of chemotherapy in HSCC. The incomplete separation of samples such as FYY and WZM indicated that other factors affected the expression profiles (Figures 1C, 1D).

Among these detected genes, thirty-three exhibited remarkably high expression, while sixty-seven exhibited significantly low expression, with p-values $<0.05$ (Figure 2A, Table 2, and Table S3). The cluster analysis showed that patients in the chemoresistant group were clustered into a clade, with two exceptions (LJ and LFL); patients in the chemosensitive group were clustered into a clade with the exception of WZM based on the 100 DEGs (Figure 3A). Among these detected miRNAs, eight exhibited differential expression, with p-values $<0.05$; all of them exhibited remarkably low expression (Figure $2 \mathrm{~B}$ and Table 3 ). The cluster analysis showed that neither patients in the chemoresistant group nor patients in the chemosensitive group were commendably clustered into a clade based on the nine low expression DE-miRNAs (Figure 3B). This finding may be because the total number of DE-miRNAs used for the cluster analysis was too small to represent the overall miRNA expression profile between the two groups.

To further verify the results of the microarray, qRT-PCR experiments were performed to detect the expression of the top ten upregulated genes, the top ten downregulated genes, and eight DE-miRNAs. The qRT-PCR results were consistent with those from the microarray (Figure 4 and Table S4), validating the reliability of the microarray data.

Core genes and miRNAs associated with the induction of chemosensitivity. To recognize the core genes associated with the induction of chemosensitivity among the identified candidate genes and miRNAs, we constructed a Bayesian network to analyze the probabilistic relationships between the candidate DEGs or DE-miRNAs and symptoms. Bayesian network analysis revealed five genes that are directly associated with chemosensitivity among the candidate DEGs (Figure 5A), namely, GAGE7, HOXC8, WARS2, HSPB8, and C160RF73. The results showed that the low expression of C16ORF73 may cause the chemosensitivity that further leads to the low expression of the HSPB8 gene and the high expression of the GAGE7, HOXC8, and WARS2 genes. It is worth noting that among these five genes, GAGE7 was the most upregulated, and HSPB8 was the most downregulated, which may indicate that GAGE7 and HSPB8 are closely related to the chemosensitivity. In addition, Bayesian network analysis revealed a tripartite network consisting of 9 DE-miRNAs, in which HBII-85-29 and hsa-miR-3937 may be directly associated with the chemosensitivity. The low expression of HBII-85-29 may cause the chemosensitivity and further lead to the low expression of hsa-miR-3937 (Figure 5B). These two miRNAs may be the core miRNAs involved in the chemosensitivity in HSCC.

Although the result is not identical, the random forest analysis also showed that the HSPB8 and C16ORF73 genes had the highest scores among the top 30 genes (Figure 6A), which emphasizes the importance of HSPB8 and C16ORF73 in the induction of chemosensitivity. Similarly, the random forest analysis revealed that HBII-85-29 and hsa-miR-3937 occupy the first and third places, respectively (Figure 6B). Bayesian network and random forest analyses suggested the significance of HBII-85-29, hsa-miR-3937, HSPB8 and C16ORF73 in induced chemosensitivity through different algorithms.
A

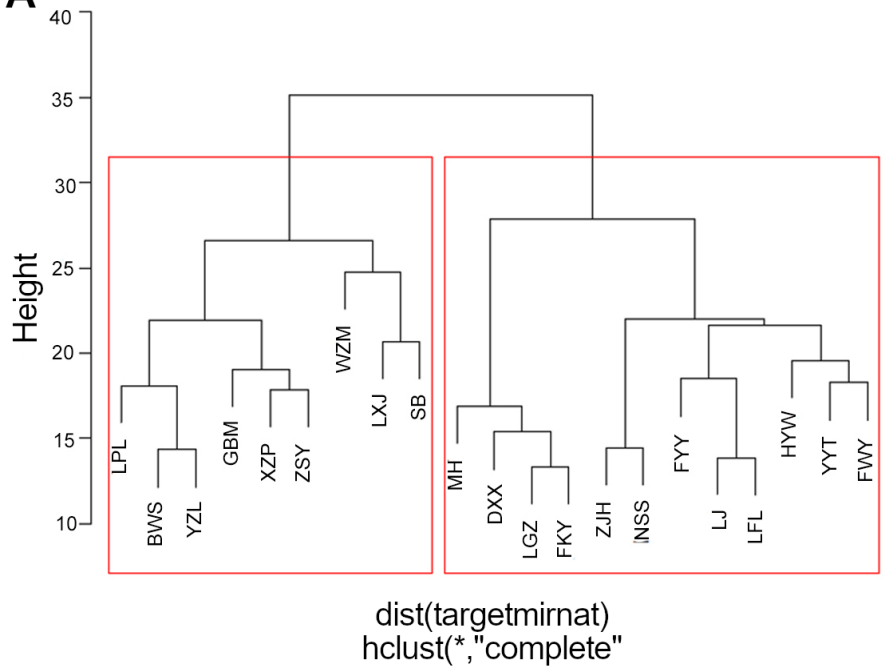

B

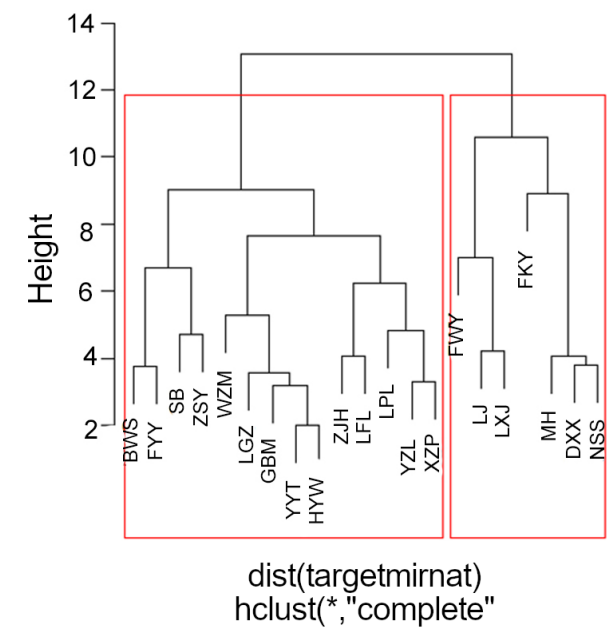

Figure 3. The distribution of differentially expressed genes and miRNAs in the two groups. A) Cluster analysis of the differently expressed genes. B) Cluster analysis of the differently expressed miRNAs. 

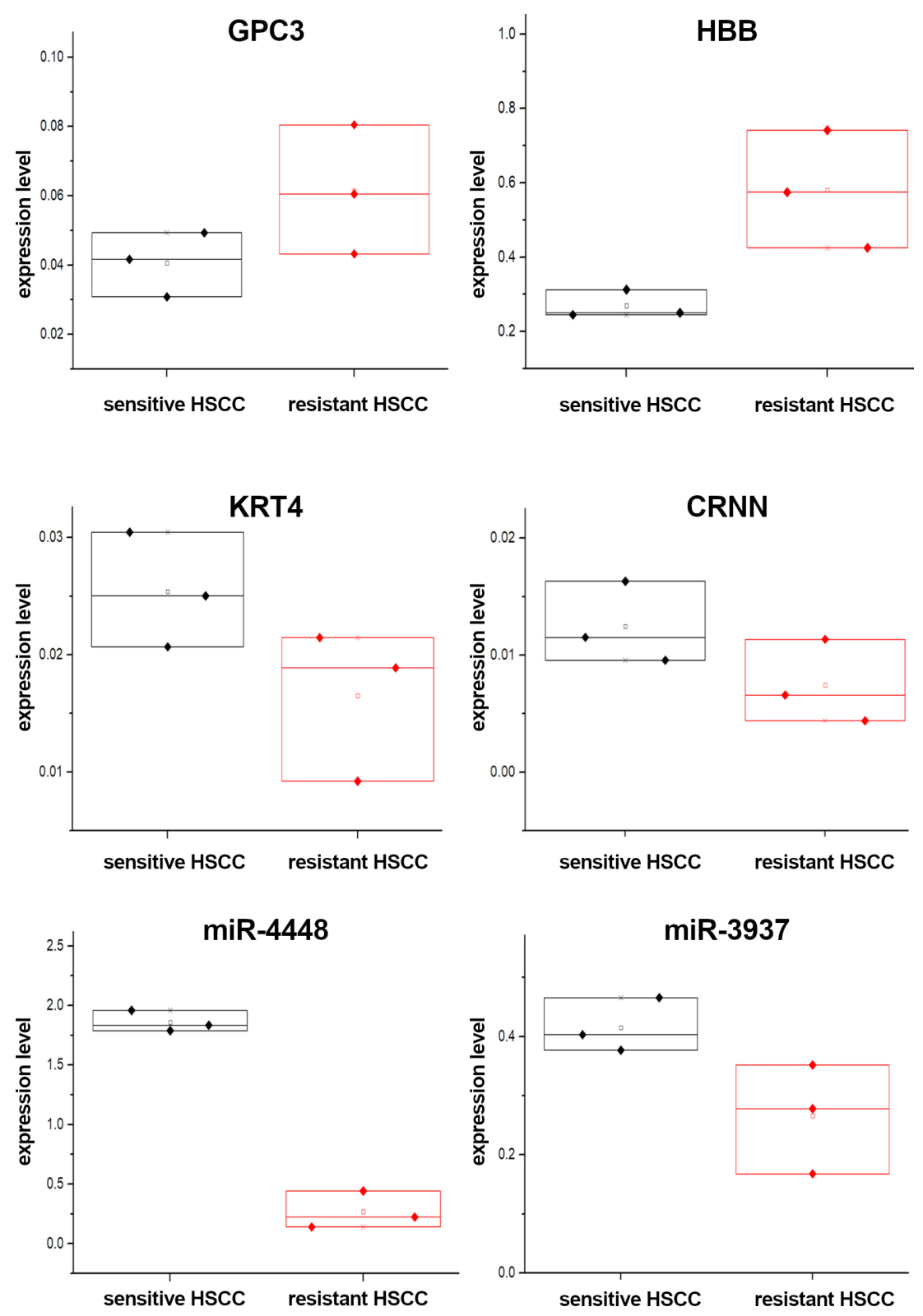

Figure 4. qRT-PCR demonstrated the expression levels of GPC3, HBB, KRT4, CRNN (all normalized to GAPHD), miR-4448 and miR-3937 (both normalized to U6) in the samples.

Integrated analysis of the mRNA and miRNA microarray profiles. Although a microarray or chip analysis is widely used to systematically investigate potentially important molecules or regulators in cancer, an integrated analysis of the relationship between the genes and miRNAs has not been performed. To analyze the roles of these differentially expressed genes and miRNAs more comprehensively, we used these DEGs and DE-miRNAs to construct a Bayesian network. Finally, an integrated regulatory network, including 8 genes and 2 miRNAs leading to chemotherapy sensitivity was constructed, and the network suggested that CCL4L1 may be a potential molecular marker for HSCC chemotherapy induction among these differentially expressed genes and miRNAs. Excess of CCL4L1 leads to the upregulation of PRAME and the downregulation of miR-375, thus decreasing HSPB8 expression and inducing chemosensitivity (Figure 7). In addition, the excess of CCL4L1 and upregulated PRAME may also result in the low expression of miR-617, leading to the aberrant expression of CEACM7, CNFN, and ODC1. In this 10-component regulatory network, the CCL4L1/ PRAME/his-miR-375/HSPB8 signaling axis plays a core role in chemotherapy sensitivity. 


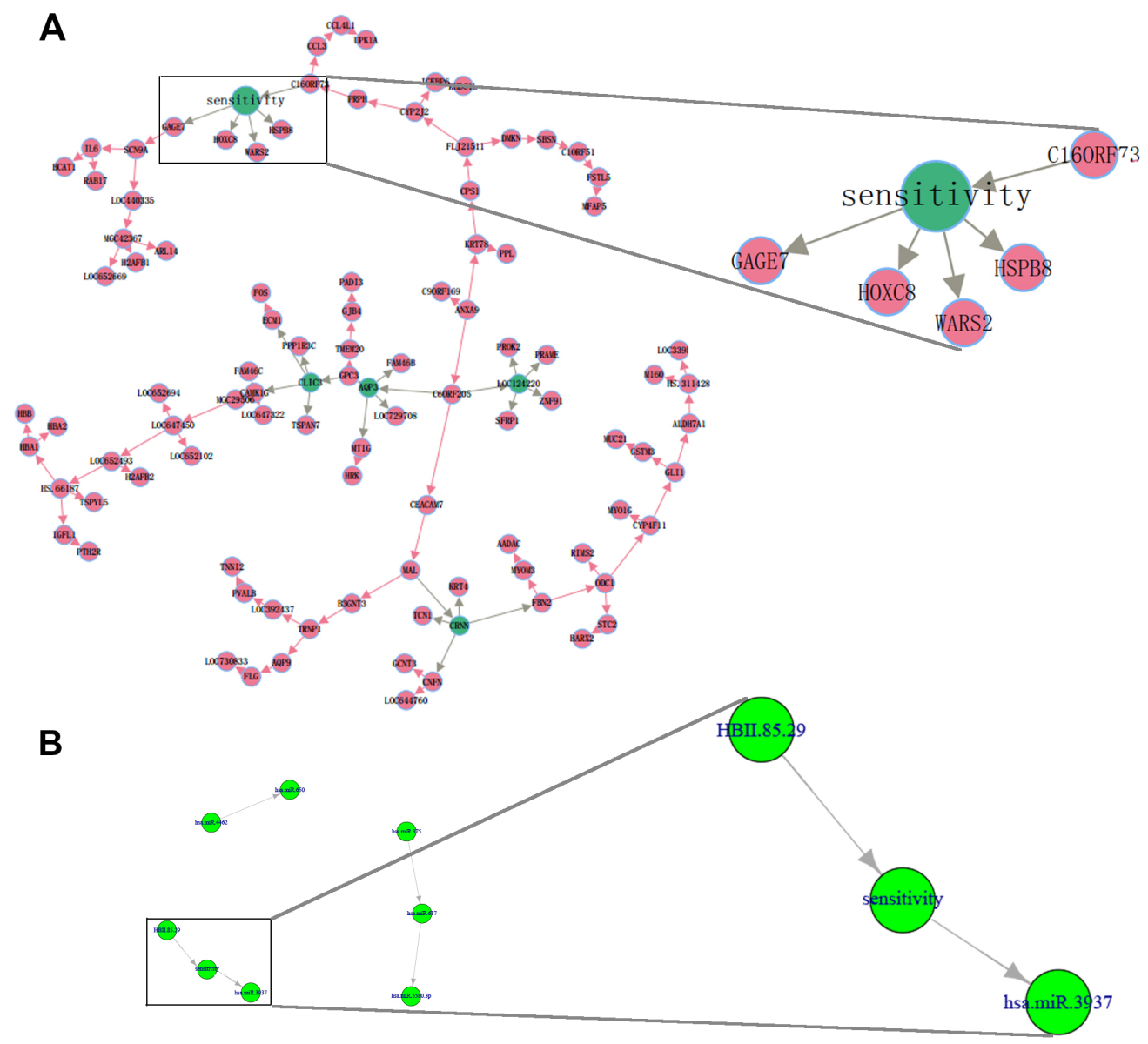

Figure 5. Bayes analysis of the core genes (A) and core miRNAs (B).

\section{Discussion}

MicroRNAs (miRNAs) comprise small (approximately $22 \mathrm{nt}$ ), endogenous RNA molecules that usually act as gene expression regulators at the posttranscriptional level in both animals and plants [23], playing an extensive role in many cellular processes, such as proliferation, metastasis, and apoptosis [24, 25]. A number of studies have shown that miRNAs play pivotal roles in tumorigenesis and the progression of cancer [26]. Abnormal miRNA regulation has been reported to affect proliferation, migration or prognosis in HSCC by targeting key proteins; for example, miRNA-34a, an important antiapoptotic gene, can affect the appearance of HSCC by regulating survival [18]; miR-370 can suppress HSCC by targeting FoxM1 [27]; and miR-21 [28], miR-155 [29] and miR-19a [26] can influence migration, invasion, and apoptosis in HSCC. These findings enrich the theoretical basis of the mechanism for the occurrence and development of HSCC. However, reports focusing on the mechanism of chemotherapy resistance in HSCC are insufficient. In our study, we analyzed the different miRNA profiles between the two groups of chemotherapy-sensitive and chemotherapyresistant samples with a microarray and then used the Bayesian network and random forest analyses to identify the two potential miRNAs most related to chemotherapy sensitivity, HBII-85-29, and hsa-miR-3937.

A number of studies have suggested that genes and miRNAs play a key role in the processes of tumorigenesis and development $[27,30]$. With developments in sequencing technologies, microarray and chip analyses can be widely used to systematically investigate potentially important molecules or regulators in cancer. For example, eight genes were associated with tumorigenesis, and RPN2 and EIF3A were associated with regional lymph node metastasis in HSCC based on the mRNA microarray analysis [31]. Sun et al. discovered 38 DE-miRNAs in supraglottic HSCC with a microRNA microarray analysis [28]. The key molecules were not isolated but interacted with each other as a whole to influence the response of cells to chemotherapy drugs.

However, an integrated analysis of the relationship between genes and miRNAs, particularly those involved in chemotherapy sensitivity in HSCC, has not been performed. 


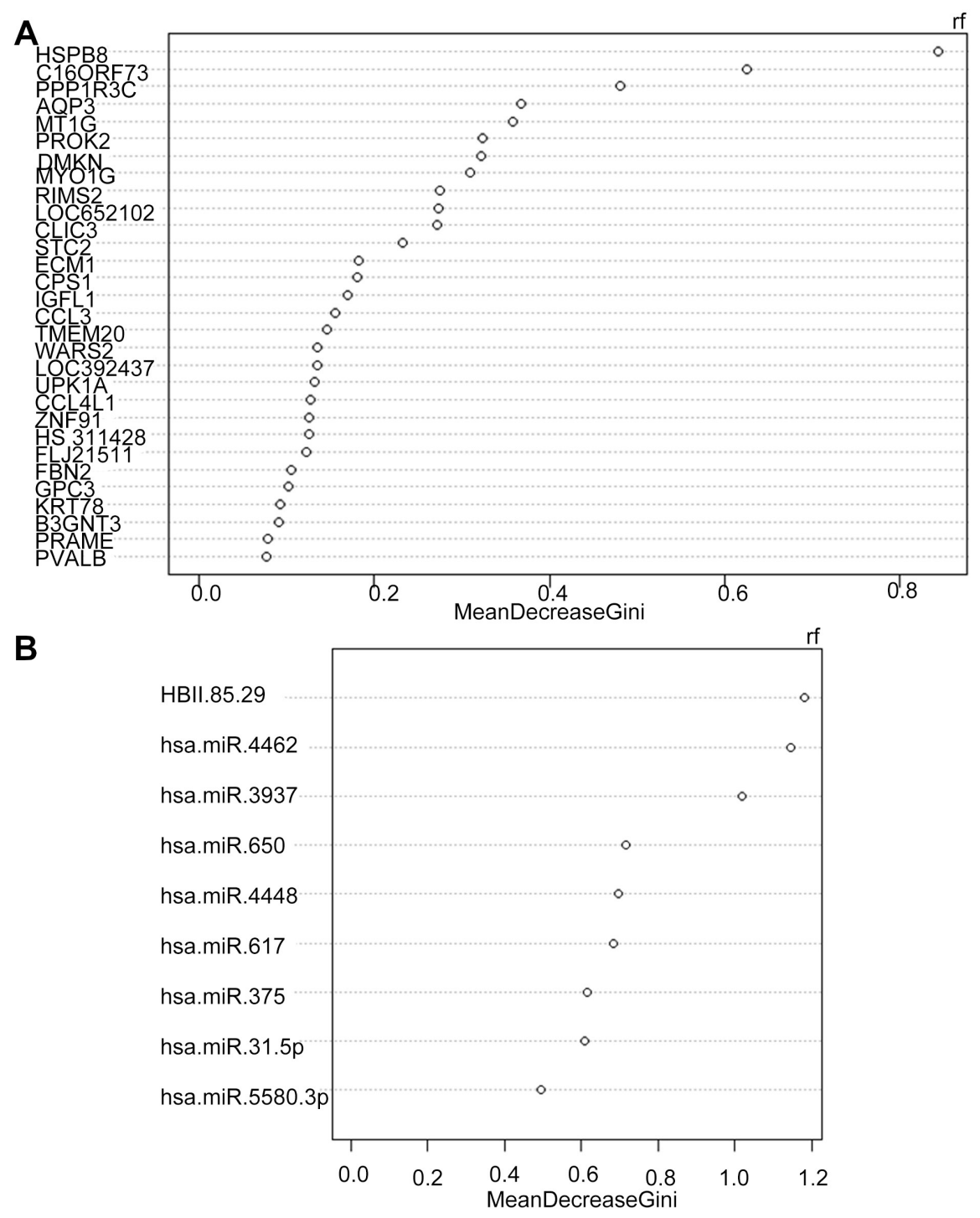

Figure 6. Random forest analysis of the DEGs (A) and DE-miRNAs (B).

In our study, we carried out microarray analysis of genes and miRNAs with chemotherapy-sensitive and chemotherapy-resistant HSCC samples. In total, 100 DEGs and nine DE-miRNAs were identified between the two groups. After investigating the expression profiles of the genes and miRNAs identified by the microarrays, cluster and random forest analyses of the identified DEGs and DE-miRNAs, respectively, were carried out. Furthermore, the relationship between the DEGs, DE-miRNAs, and chemotherapy sensitivity was fully excavated by the Bayesian network. Finally, the Bayesian analysis showed an integrated regulatory network including eight genes and two miRNAs, in which redundant CCL4L1 leads to the upregulation of PRAME and the downregulation of miR-375, thus decreasing the HSPB8 expression and inducing chemosensitivity. This network implies that CCL4L1 may be a pivotal molecule for the induction of the HSCC chemotherapy sensitivity, and CCL4L1 as well as PRAME, miR-375, and HSPB8 are expected to be potential therapeutic targets and diagnostic markers after further experimental support.

The small heat shock protein B8 (HSPB8) belongs to the superfamily of small heat shock proteins that contain a conservative alpha-crystallin domain at the C-terminus. HSPB8 is believed to be associated with the regulation of cell apoptosis, proliferation, and carcinogenesis. For example, HSPB8 accelerates cancer cell growth by activating the ERK-CREB pathway and is indicative of a poor prognosis in gastric cancer patients [32]. HSPB8 reduces the migration of hepatocellular carcinoma cells by suppressing the phosphoinositide 3-kinase (PI3K)/AKT pathway [33]. To 


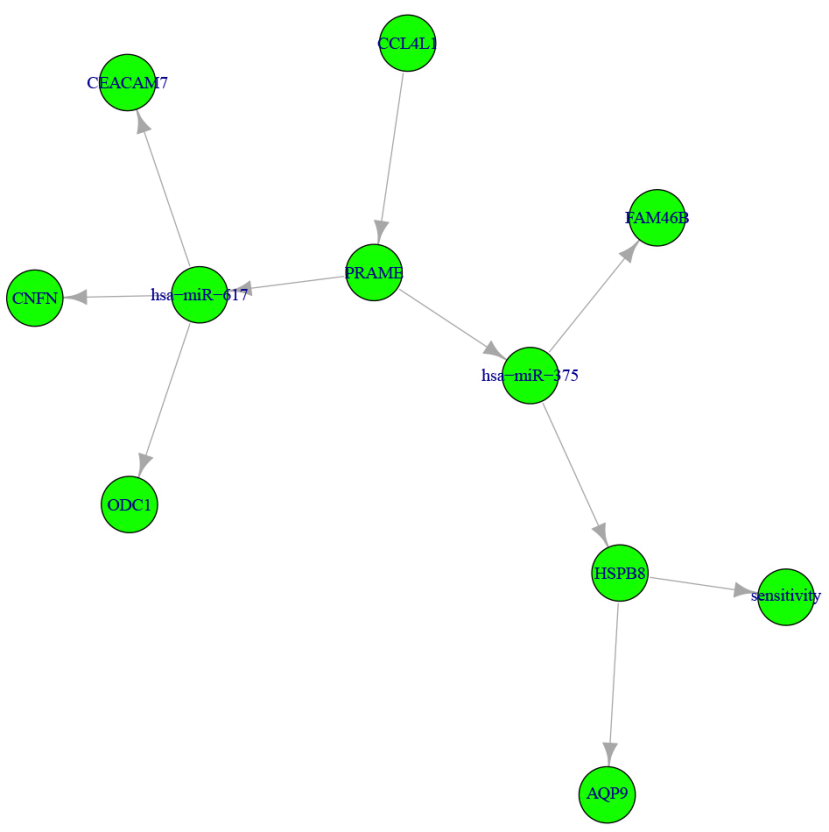

Figure 7. Integrated analysis of the DEGs and DE-miRNAs. Integrated analysis of the mRNA and miRNA microarray profiles revealed a PRAME/miR375/HSPB8 signal axis that contributes to chemosensitivity.

date, there is no literature linking HSPB8 to HSCC or sensitivity. Our results showed that the expression of HSPB8 is reduced in chemosensitive HSCC samples, and subsequent Bayesian network and random forest analyses showed that HSPB8 plays an important role in the induction of chemosensitivity. These findings revealed, for the first time, the association between HSPB8 and HSCC chemosensitivity and enriched the mechanism and therapeutic targets of HSCC chemotherapy sensitivity.

CCL4L1 and CCL3L1 are the analogs of chemokines CCL4 and CCL3, respectively, which are similar in function, and may also play an important role in the acute rejection of organ transplantation and the infection of hepatitis B virus [34]. Recent studies have found that the CCL4L1 gene has copy number polymorphisms, and carriers with high copy numbers often exhibit high levels of transcription and protein secretion [35-37]. Currently, no data suggest that CCL4L1 is related to HSCC. Our analysis, for the first time, showed that the expression of CCL4L1 in chemotherapy-resistant samples was significantly higher than that in chemotherapy-sensitive samples, and subsequent bioinformatics analysis showed that CCL4L1 was a key inducing factor for chemotherapy sensitivity. It is worth exploring whether the high expression of CCL4L1 is related to the high copy number and its effect on chemotherapeutic impact in HSCC. These findings contribute to a deeper understanding of the potential mechanisms of HSCC chemotherapy resistance. In addition, our study provides a theoretical foundation for further experimental studies exploring therapeutic targets and diagnostic markers.
Supplementary information is available in the online version of the paper.

Acknowledgements: This study was supported by grants from the Beijing Natural Science Foundation Program and Scientific Research Key Program of Beijing Municipal Commission of Education (KZ201910025034), the Beijing Municipal Administration of Hospitals' Ascent Plan (DFL20180202), the Capital Health Research and Development of Special (No. 2018-2-2054), the Beijing Municipal Administration of Hospitals Incubating Program (PX2018009), and the Beijing Administration of Traditional Chinese Medicine (QN2018-32).

\section{References}

[1] SIEGEL RL, FEDEWA SA, MILLER KD, GODING-SAUER A, PINHEIRO PS et al. Cancer statistics for Hispanics/Latinos, 2015. CA Cancer J Clin 2015; 65: 457-480. https://doi. org/10.3322/caac. 21314

[2] RAMROTH H, SCHOEPS A, RUDOLPH E, DYCKHOFF G, PLINKERT $P$ et al. Factors predicting survival after diagnosis of laryngeal cancer. Oral Oncol 2011; 47: 1154-1158. https://doi.org/10.1016/j.oraloncology.2011.08.003

[3] LIGIER K, BELOT A, LAUNOY G, VELTEN M, BOSSARD $\mathrm{N}$ et al. Descriptive epidemiology of upper aerodigestive tract cancers in France: incidence over 1980-2005 and projection to 2010. Oral Oncol 2011; 47: 302-307. https://doi. org/10.1016/j.oraloncology.2011.02.013

[4] TAKES RP, STROJAN P, SILVER CE, BRADLEY PJ, HAIGENTZ M JR et al. Current trends in initial management of hypopharyngeal cancer: the declining use of open surgery. Head Neck 2012; 34: 270-281. https://doi. org/10.1002/hed.21613 
[5] MARIONI G. Letter to the editors: Essentials for an updated epidemiology of laryngeal carcinoma. Cancer Treat Rev 2012; 38: 559. https://doi.org/10.1016/j.ctrv.2012.02.005

[6] HOFFMAN HT, KARNELL LH, SHAH JP, ARIYAN S, BROWN GS et al. Hypopharyngeal cancer patient care evaluation. Laryngoscope 1997; 107: 1005-1017. https://doi. org/10.1097/00005537-199708000-00001

[7] GODBALLE C, JORGENSEN K, HANSEN O, BASTHOLT L. Hypopharyngeal cancer: results of treatment based on radiation therapy and salvage surgery. Laryngoscope 2002; 112: 834-838. https://doi.org/10.1097/00005537-20020500000011

[8] ZAFEREO M. Surgical salvage of recurrent cancer of the head and neck. Curr Oncol Rep 2014; 16: 386. https://doi. org/10.1007/s11912-014-0386-0

[9] GAMBARDELLA C, POLISTENA A, SANGUINETTI A, PATRONE R, NAPOLITANO $S$ et al. Unintentional recurrent laryngeal nerve injuries following thyroidectomy: Is it the surgeon who pays the bill? Int J Surg 2017; 41 Suppl 1: S55-S59. https://doi.org/10.1016/j.ijsu.2017.01.112

[10] THOMPSON LD. Laryngeal Dysplasia, Squamous Cell Carcinoma, and Variants. Surg Pathol Clin 2017; 10: 15-33. https://doi.org/10.1016/j.path.2016.10.003

[11] ROBBINS KT. The evolving role of combined modality therapy in head and neck cancer. Arch Otolaryngol Head Neck Surg 2000; 126: 265-269. https://doi.org/10.1001/archotol.126.3.265

[12] POINTREAU Y, GARAUD P, CHAPET S, SIRE C, TUCHAIS C et al. Randomized trial of induction chemotherapy with cisplatin and 5-fluorouracil with or without docetaxel for larynx preservation. J Natl Cancer Inst 2009; 101: 498506. https://doi.org/10.1093/jnci/djp007

[13] SINNBERG T, MENZEL M, EWERTH D, SAUER B, SCHWARZ $M$ et al. beta-Catenin signaling increases during melanoma progression and promotes tumor cell survival and chemoresistance. PLoS One 2011; 6: e23429. https://doi. org/10.1371/journal.pone.0023429

[14] LI J, WOOD WH 3RD, BECKER KG, WEERARATNA AT, MORIN PJ. Gene expression response to cisplatin treatment in drug-sensitive and drug-resistant ovarian cancer cells. Oncogene 2007; 26: 2860-2872. https://doi.org/10.1038/ sj.onc. 1210086

[15] WEI S, WANG Y, XU H, KUANG Y. Screening of potential biomarkers for chemoresistant ovarian carcinoma with miRNA expression profiling data by bioinformatics approach. Oncol Lett 2015; 10: 2427-2431. https://doi.org/10.3892/ ol.2015.3610

[16] DAI F, ZHANG Y, ZHU X, SHAN N, CHEN Y. The antichemoresistant effect and mechanism of MUC1 aptamermiR-29b chimera in ovarian cancer. Gynecol Oncol 2013; 131: 451-459. https://doi.org/10.1016/j.ygyno.2013.07.112

[17] SAEED AI, SHAROV V, WHITE J, LI J, LIANG W et al. TM4: a free, open-source system for microarray data management and analysis. Biotechniques 2003; 34: 374-378. https://doi.org/10.2144/03342mt01
[18] SMYTH GK. Linear models and empirical bayes methods for assessing differential expression in microarray experiments. Stat Appl Genet Mol Biol 2004; 3: Article3. https:// doi.org/10.2202/1544-6115.1027

[19] RAHMATALLAH Y, ZYBAILOV B, EMMERT-STREIB F, GLAZKO G. GSAR: Bioconductor package for Gene Set analysis in R. BMC Bioinformatics 2017; 18: 61. https://doi. org/10.1186/s12859-017-1482-6

[20] BRONSTEIN AM, BRONSTEIN MM, KIMMEL R. Generalized multidimensional scaling: a framework for isometry-invariant partial surface matching Proc Natl Acad Sci U S A 2006; 103: 1168-1172. https://doi.org/10.1073/ pnas. 0508601103

[21] WAHDE M, HERTZ J. Coarse-grained reverse engineering of genetic regulatory networks. Biosystems 2000; 55: 129136. https://doi.org/10.1016/s0303-2647(99)00090-8

[22] BREIMAN L. Random Forests. Machine Learning 2001; 45: $5-32$.

[23] BARTEL DP. MicroRNAs: target recognition and regulatory functions. Cell 2009; 136: 215-233. https://doi.org/10.1016/j. cell.2009.01.002

[24] HE L, HANNON GJ. MicroRNAs: small RNAs with a big role in gene regulation. Nat Rev Genet 2004; 5: 522-531. https://doi.org/10.1038/nrg1379

[25] CUI Y, HAN J, XIAO Z, CHEN T, WANG B et al. The miR-20-Rest-Wnt signaling axis regulates neural progenitor cell differentiation. Sci Rep 2016; 6: 23300. https://doi. org/10.1038/srep23300

[26] IORIO MV, VISONE R, DI LEVA G, DONATI V, PETROCCA $F$ et al. MicroRNA signatures in human ovarian cancer. Cancer Res 2007; 67: 8699-8707. https://doi. org/10.1158/0008-5472.CAN-07-1936

[27] CALIN GA, CROCE CM. MicroRNA signatures in human cancers. Nat Rev Cancer 2006; 6: 857-866. https://doi. org/10.1038/nrc1997

[28] SUN X, SONG Y, TAI X, LIU B, JI W. MicroRNA expression and its detection in human supraglottic laryngeal squamous cell carcinoma. Biomed Rep 2013; 1: 743-746. https://doi. org/10.3892/br.2013.143

[29] JIANG Y, ZHANG R, LV H, LI J, WANG M et al. HGPGD: the human gene population genetic difference database. PLoS One 2013; 8: e64150. https://doi.org/10.1371/journal. pone.0064150

[30] FUTREAL PA, COIN L, MARSHALL M, DOWN T, HUBBARD $T$ et al. A census of human cancer genes. Nat Rev Cancer 2004; 4: 177-183. https://doi.org/10.1038/nrc1299

[31] LIAN M, FANG J, HAN D, MA H, FENG L et al. Microarray gene expression analysis of tumorigenesis and regional lymph node metastasis in laryngeal squamous cell carcinoma. PLoS One 2013; 8: e84854. https://doi.org/10.1371/ journal.pone.0084854

[32] SHEN J, LI M, MIN L. HSPB8 promotes cancer cell growth by activating the ERKCREB pathway and is indicative of a poor prognosis in gastric cancer patients. Oncol Rep 2018; 39: 2978-2986. https://doi.org/10.3892/or.2018.6376 
[33] MATSUSHIMA-NISHIWAKI R, TOYODA H, TAKAMATSU R, YASUDA E, OKUDA $S$ et al. Heat shock protein 22 (HSPB8) reduces the migration of hepatocellular carcinoma cells through the suppression of the phosphoinositide 3-kinase (PI3K)/AKT pathway. Biochim Biophys Acta Mol Basis Dis 2017; 1863: 1629-1639. https://doi.org/10.1016/j. bbadis.2017.04.021

[34] HANCOCK WW. Chemokine receptor-dependent alloresponses. Immunol Rev 2003; 196: 37-50. https://doi. org/10.1046/j.1600-065x.2003.00084.x

[35] MODI WS. CCL3L1 and CCL4L1 chemokine genes are located in a segmental duplication at chromosome $17 \mathrm{q} 12$. Genomics 2004; 83: 735-738. https://doi.org/10.1016/j.ygeno.2003.09.019
[36] COLOBRAN R, PEDROSA E, CARRETERO-IGLESIA L, JUAN M. Copy number variation in chemokine superfamily: the complex scene of CCL3L-CCL4L genes in health and disease. Clin Exp Immunol 2010; 162: 41-52. https://doi. org/10.1111/j.1365-2249.2010.04224.x

[37] GRUNHAGE F, NATTERMANN J, GRESSNER OA, WASMUTH HE, HELLERBRAND C et al. Lower copy numbers of the chemokine CCL3L1 gene in patients with chronic hepatitis C. J Hepatol 2010; 52: 153-159. https://doi. org/10.1016/j.jhep.2009.11.001 